\title{
Assessment of the Midwives Knowledge on Utilization of Partograph in Management of Labour in Public Health Facilities in Tharaka Nithi County
}

\author{
Mwari P.S ${ }^{1}$, Gitonga LK ${ }^{2}$, Mukhwana E.S ${ }^{3}$ \\ ${ }^{1,2,3}$ Department of Nursing, Chuka University, P.O Box 109-60400, Chuka, Kenya \\ Corresponding Author: Mwari P.S
}

\begin{abstract}
The World Health Organization (WHO) has recommended Partograph as a labor management tool due to its impact in reducing obstetric labor complications and maternal deaths over the years. This labor management tool is inexpensive and appropriate for use in low-resource settings, particularly in developing countries. Despite the fact that many African countries, including Ethiopia, Kenya, and Nigeria, have adopted the Partograph in labor management, there is little information on midwives' knowledge on the use of the tool in labor management. Understanding of how to use a Partograph is essential for reducing complications and maternal deaths. The purpose of this study was to determine midwives' knowledge on using the Partograph in labor management. The study adopted a cross sectional survey design. 45 midwives were randomly selected from 16 public health facilities in Tharaka Nithi County. Questionnaires were used to collect data. Focused Group Discussions were also conducted with 77 postnatal mothers who gave birth in the 16 health facilities. The collected data was analyzed using descriptive statistics (means, frequencies, and percentages) and Chi-Square tests. Thematic analysis was used to examine qualitative data. Results showed that $74 \%$ of midwives had used Partograph in labor monitoring, while $26 \%$ had never used the tool. $60 \%$ of the midwives had received Partograph training. Despite not having been trained, some midwives were using the Partograph. Findings revealed inconsistencies in the use of Partograph in labor management. A large proportion of midwives deviated from the WHO's recommended use of Partograph in the first stage of labor. The study recommended that the Tharaka Nithi County government, through the Ministry of Health, invest in Partograph utilization in-service training programs to provide nurses and midwives working in the maternity wing the necessary knowledge and skills for proper Partograph utilization. There is also a need for policy changes in institutions to ensure that nurses use of the Partograph as a strategy for reducing maternal mortality rates, improving motherhood, and labor management in the County.
\end{abstract}

Key Words: Partograph; labour management; public health facilities.

\section{INTRODUCTION}

Globally, the Maternal Mortality Rates (MMR) are still high mainly due to obstetric labor complications [1]. Despite efforts to promote safe maternal care, maternal deaths have not reduced in recent years. The World Health Organization (WHO) estimates indicate that there were 830 maternal deaths per day due to pregnancy-related complications in the year
2014 with developing countries reporting approximately 5.7 million perinatal deaths each year [2]. These global trends prompted health scientists and researchers to look into strategies to reduce pregnancy-related complications and maternal mortality rates. The WHO recommended the use of a labor management tool, the Partograph, in 2011 to monitor and evaluate women during their first stage of labor and to provide 
appropriate interventions to avoid any obstetric complications [3]. Healthcare providers use this tool to make labor management decisions based on a visual overview (2011). Kenya is one of the developing countries that has adopted the WHO's recommendation to use the Partograph in maternal healthcare to reduce MMR. Despite its utility in labor management, Partograph utilization in the country remains low [4].

Over the years, the Kenyan Government has increased their investments in labor room management and quality labor room practices in order to improve neonatal and maternal health. This is because of the risks associated with childbirth, birth and labor management. The goal here is to achieve Global Sustainable Development Goal 3, which advocates for improved maternal care and reduced maternal mortality.

The Partograph is one of the universal labor management tools because of its cost-effectiveness and suitability in low-resource settings common in primary care health facilities. Despite partograph's widespread use and advocacy over the years, cases of obstructed labor continue to be reported, indicating inefficiency in labor monitoring by obstetric caregivers who use the tool [5]. Studies reveal that a significant proportion of healthcare providers working in labor units in various hospitals across Africa have poor attitude on Partograph use. Yisma investigated Partograph utilization in Ethiopia and discovered that, while many midwives reported knowing how to use the tool, its use in labor management in health centers and hospitals remained low at 67.9 percent and 34.3 percent, respectively [6].

In addition, research has revealed a knowledge gap in the use of Partograph in labor management. For example, a study that looked at the knowledge levels of Partograph use among midwives and other staff in delivery units in healthcare facilities in Nigeria's Delta region discovered that Partograph was underutilized in labor units because healthcare providers lacked adequate knowledge on how to use the tool
[7]. In Rwanda, Kalisa, conducted a study on Partograph utilization and discovered that despite training, the majority (76\%) of healthcare workers had difficulty using the tool [8]. A similar study conducted in Nigeria produced sufficient evidence to support the findings on poor knowledge on the use of Partograph in the country [9].

Kenya's healthcare system is one of those in African countries that has transitioned from paper-based to erecording in labor management. However, not all health care facilities are linked to the electronic medical record system. Furthermore, most health-care facilities lack modern equipment such as ultrasound machines, which can accurately and instantly monitor and record labor. The MMR is still high at 342/100000much higher than the SDG target of 155/100000 (4; 15). The Partograph is thus still invaluable in Kenya, as it is in other SubSaharan African countries, but nurses and midwives continue to face challenges in using it, despite Partograph utilization being taught in medical colleges and other institutions of higher learning [10].

Training health workers on the use of Partograph promotes safe motherhood and is one of the MMR-lowering strategies. A study conducted in Western Kenya in the early 2000s/early 2001 on the impact of training on Partograph utilization in the improvement of maternal care found a significant improvement in maternal care [11]. Findings showed that 85 percent of the health facilities used Partograph after training, compared to 11 percent before training. However, there are still many gaps in knowledge and poor attitudes toward the adoption and use of the Partograph. In a different study, [10] concluded that the Kenyan health system had challenges with inadequate knowledge and incorrect use of Partograph use. The study, which took place at Moi Referral and Teaching Hospital in Kenya, looked at 234 Partograph recordings in labor management. The findings indicated that all the Partograph were either incorrectly filled or were incomplete. 
Despite the Country's strong emphasis on its use, standardization of Partograph utilization remains a concern. This study looked at midwives' knowledge of Partograph utilization in public health facilities in Tharaka Nithi County, as well as its impact on labor management and maternal care. Tharaka Nithi County still has a MMR of 365/100000 with many public health facilities lacking ultra-modern equipment to instantly monitor labor.

\section{MATERIALS AND METHOD}

The study used a cross-sectional survey design to provide information about mid wives' exposure to the Partograph as well as its utilization [12]. The design was adopted due to its cost-effectiveness and wide application in public health planning for estimating intervention outcome prevalence. The design was also used to evaluate midwives' use of Partograph in TNC's health facilities labor management.

Using questionnaires and Focus Group Discussions, data was collected from 16 health facilities in the County that had a maternity wing. A pretest study had been conducted in Embu County to ascertain the validity and reliability of the instruments. For this study, a sample size of $10-30 \%$ was deemed appropriate and used (Orodho 2008). 20\% of the County's midwives (45) were selected randomly. From the postnatal care services postnatal a $20 \%$ sample mothers (77) was also randomly selected

To examine midwives' knowledge levels for Partograph use, both qualitative and quantitative data analysis methods were used. Responses to knowledge items on the research tool were scored, and Chi square tests were used to establish associations of Midwives' knowledge with utilization on Partograph in Tharaka Nithi County public health facilities. Data from postnatal mothers were gathered through Focused Group discussions. The Statistical Package for Social Sciences (SPSS) version 22 software was used for quantitative data analysis. Descriptive statistics including means, standard deviations, and frequencies were computed, and results presented in form of narratives, tables, and graphs. Qualitative data was analyzed thematically.

The Chuka University Ethical Review Committee and the National Commission for Science, Technology, and Innovation (NACOSTI) granted permission to conduct the study. The researcher also obtained permission from each hospital's administration to collect data. To ensure that respondents were autonomous in their decision to participate in the study, each participant was approached individually. The study sought, obtained, and used informed consent and debriefing for all study participants.

\section{RESULT}

The response rate was $100 \%$ with all the 45 respondents returning their filled questionnaires. The researcher also conducted eight focus group discussions, with each group having 10 mothers.

Table 1: Response Rate for research Instruments

\begin{tabular}{|l|l|l|}
\hline \multirow{2}{*}{ Instrument Category } & Response \\
\cline { 2 - 3 } & Frequency & Percentage \\
\hline Questionnaires & 45 & $100 \%$ \\
\hline Focused Group Discussion & 8 & $100 \%$ \\
\hline Total & 40 & 100 \\
\hline
\end{tabular}

\section{Respondent's Age Distribution}

The majority of the respondents (62\%) were between 30 to 40 years with those aged between 50-60 years at (7\%), 4050 years at $18 \%$ and those of $20-30$ years at $13 \%$.

\section{Respondent's Gender}

Majority of the Midwives were females (71\%)

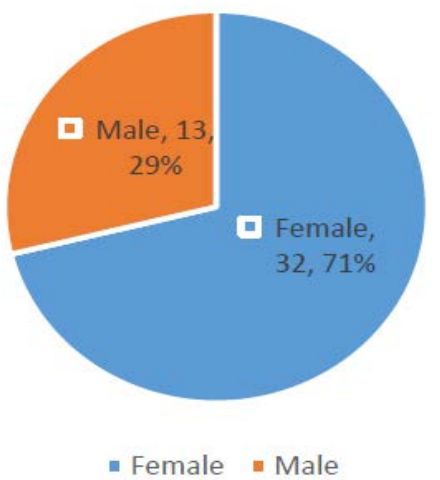

Figure 1: Gender distribution of the Midwives 

public health facilities in Tharaka Nithi County.

\section{Professional qualification}

The results on the professional qualifications of the Midwives are presented in Table 2.

Table 2: Midwives' professional/educational qualifications

\begin{tabular}{|l|l|l|}
\hline Qualification & Frequency & Percentage \\
\hline Registered midwife & 6 & 17.7 \\
\hline Krchn & 34 & 75.6 \\
\hline Enrolled Nurse & 1 & 2.2 \\
\hline Bachelor Nurse & 2 & 4.5 \\
\hline Total & 45 & 100.0 \\
\hline
\end{tabular}

Majority (75.6\%) belonged to KRCHN category. The registered midwives were $17.7 \%$, those with bachelors of nursing and enrolled nurse wee at $4.5 \%$ and $2.2 \%$, respectively. The results revealed that the public health facilities in the County had midwives who had met a midwife's minimum qualifications.

\section{Pre-service training for the midwives}

The midwives had spent different periods in their pre-service training. At the time of the study, there were no midwives in pre-service training.

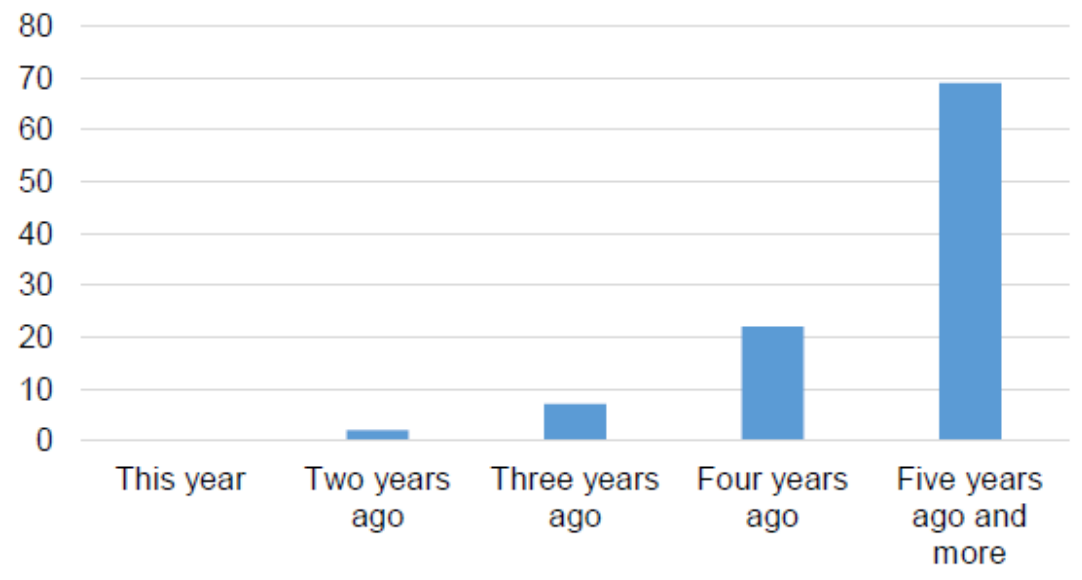

Figure 2: Duration of pre-service training for the midwives

Figure 2 shows that the majority of midwives (69\%) had completed their preservice training between five and more years before the data was collected. $22 \%$ of the midwives completed four years ago, while seven percent and two percent completed three and two years ago, respectively.

\section{In-service training}

Results on in-service training are presented in Figure 3.

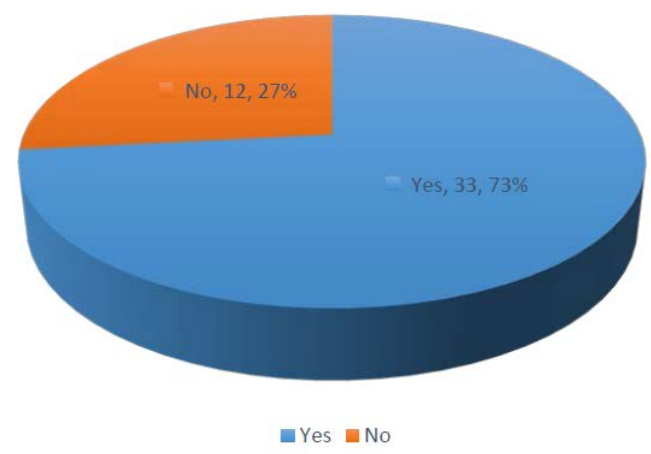

Figure 3: Proportion of midwives that received in-service training
The majority of midwives (73\%) had received training while working. The remaining $27 \%$ had received no in-service training on labor management. These findings raises concerns about the effectiveness of untrained midwives in assisting pregnant women during the labor process.

\section{Type of in-service training}

The study sought to establish the type of in-service training that the midwives received on labour management. Table 3 presents a summary of the findings

Table 3: Type of in-service training received by midwives in labour management

\begin{tabular}{|l|l|l|}
\hline Response & Frequency & Percentage \\
\hline $\begin{array}{l}\text { Emergency Obstetric and Neonatal } \\
\text { care (EmONC) }\end{array}$ & 18 & 40.0 \\
\hline Advanced Life Support in Obstetrics & 15 & 33.3. \\
\hline None of the above & 12 & 26.7 \\
\hline Total & 45 & 100 \\
\hline
\end{tabular}

The midwives reported having received two types of obstetrics training including emergency obstetric and neonatal 
care (EmONC) (40\%) and advanced life support in obstetrics (33.3\%). More than a quarter $(26.7 \%)$ of the midwives had not received any of the two forms of obstetric training.

\section{Years of experience}

Respondents' years of experience were considered important for evaluation in this study. The results are presented in figure 4 .

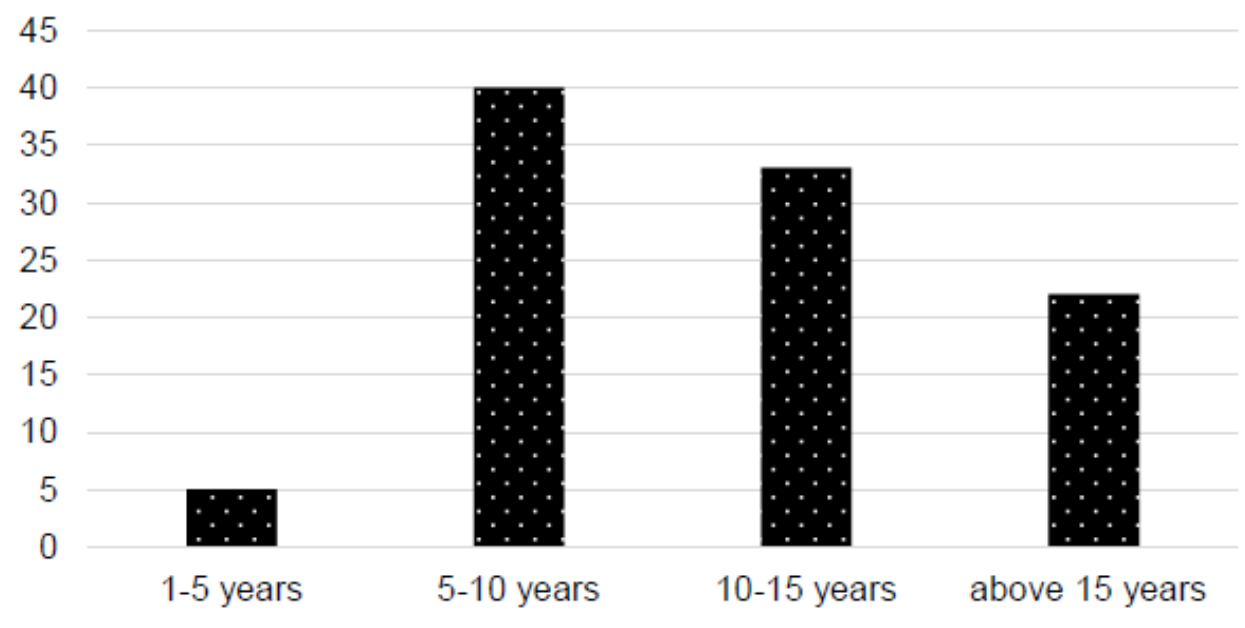

Figure 4: Respondents' working experience in years

The results showed that the majority (40\%) had 5 to 10 years of practicing labour management while $22 \%$ had over 15 years of work experience (Figure 4). Thirty-three percent of the midwives had worked for 10 to 15 years with the least percentage (5\%) having worked for five years and below. The working experience results implied that most of the midwives in the study had the immense experience to provide adequate insight and information to generate viable findings and conclusions.

\section{Ward/Unit of Practice}

The midwives in the sampled health facilities worked in different maternal units.

Table 4: Ward/Unit of Practice

Table 4: Ward/Unit of Practice
\begin{tabular}{|l|l|l|}
\hline Response & Frequency & Percentage \\
\hline Antenatal ward & 2 & 4.7 \\
\hline Labour ward & 38 & 83.2 \\
\hline Post-natal ward & 5 & 11.1 \\
\hline Total & 45 & 100.0 \\
\hline
\end{tabular}

Table 4 indicates that the majority of the midwives (83.2\%) were working in the labour wards during the time of the study while $11.1 \%$ were placed in the post-natal wards, and $4.7 \%$ were in the neonatal ward. This implies all the selected respondents had a chance to use the Partograph.

\section{Partograph Utilization}

When the midwives were asked about utilization of the Partograph, majority said they had never used the tool. The results are presented in Fig. 5.

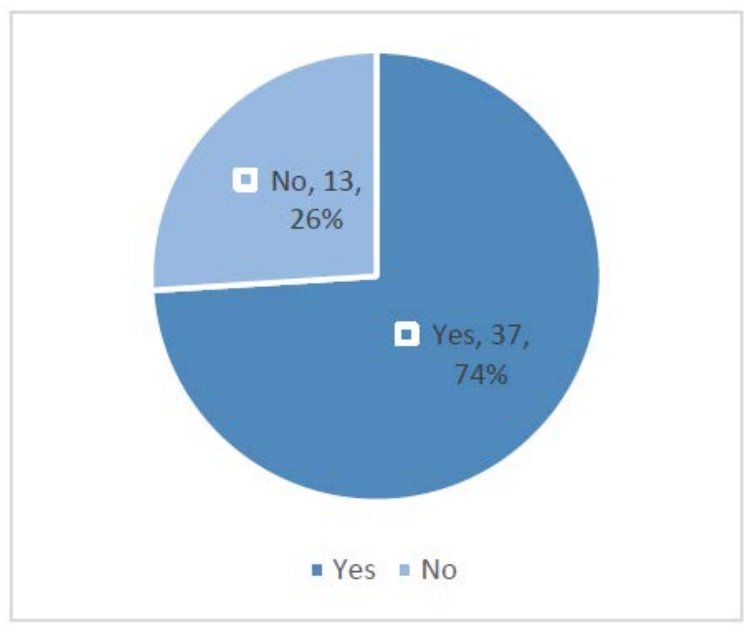

Figure 5: Midwives Use of the Partograph

The majority of labor unit midwives (74 \%) confirmed using Partograph when dealing with pregnant women during the labor process. However, more than a quarter (26\%) of the midwives said they had never used a Partograph when managing labor in the wards.

Mothers expressed concerns about midwives' basic knowledge of Partograph 
use during Focus Group Discussions. According to the mothers, only a few midwives used the labor management tool to record and assess the labor. According to the mothers, some midwives sought clarification on the interpretation of Partograph from clinical officers and doctors. This finding indicates that, while most midwives had basic knowledge of the Partograph, there was still limited knowledge of its use, including interpretation, among midwives.

\section{Definition of Partograph}

The midwives had a different way of defining the Partograph. The three definitions that the midwives were required to interpret are in the table below.

Table 5: Midwives' Definition of a Partograph

\begin{tabular}{|l|l|l|}
\hline Response & Frequency & Percentage \\
\hline $\begin{array}{l}\text { 1. A chart for labour monitoring by } \\
\text { doctors }\end{array}$ & 38 & 84.4 \\
\hline $\begin{array}{l}\text { 2. A complex tool that provides a } \\
\text { pictorial overview to be used by } \\
\text { midwives }\end{array}$ & 32 & 71.1 \\
\hline $\begin{array}{l}\text { 3. A simple graphic with the ability } \\
\text { to record labour progress and report } \\
\text { mother's and child's salient } \\
\text { conditions against time in hours }\end{array}$ & & 62.2 \\
\hline
\end{tabular}

Some midwives defined the Partograph in more than one way. The majority (84.4 \%) thought a Partograph was more like a chart used by doctors to monitor labor. $71 \%$ of midwives believed in the partograph's complexity and ability to provide a pictorial overview that aids midwives in monitoring the labor process. Other respondents (62.2\%) agreed that a Partograph is a simple graphic that records labor progress and helps monitor the mother and child's conditions over time.

\section{Institutional training on use of Partograph}

The study sought to establish if the respondents had received training on use of Partograph while in medical institutions.

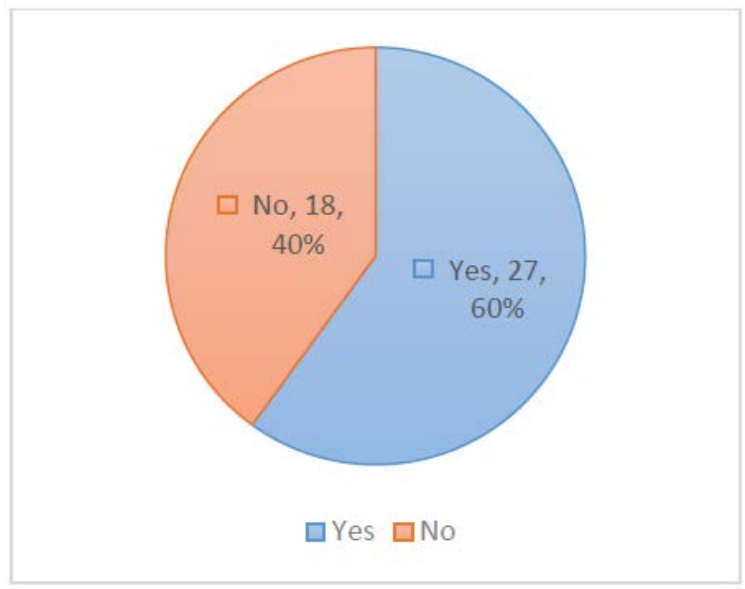

Figure 6: Percentage of midwives trained on Partograph utilization

The results on midwives who had been trained on Partograph utilization showed that $60 \%$ of them had been trained to use the labour management tool. However, the remaining $40 \%$ had not received training on the use of Partograph in managing labour. The $40 \%$ of the midwives who lacked training on Partograph use raised concern in labour management in TNC.

When questioned further, the $40 \%$ who did not receive college training stated that they were trained by colleagues, workshops, or pre-service training, and in some cases all of them.

\section{Place of training on Partograph Utilization}

The researcher was interested in finding out where the trained midwives had received their training. A majority (77.8\%) said that a colleague had trained them while $57.8 \%$ received

Table 6: Place of training on Partograph Utilization

\begin{tabular}{|l|l|l|}
\hline Response & Frequency & Percentage \\
\hline From a colleague & 35 & 77.8 \\
\hline From workshop & 26 & 57.8 \\
\hline From pre-service training & 21 & 46.7 \\
\hline
\end{tabular}

\section{Knowledge on the use of the Partograph}

The researcher went further to investigate midwives' knowledge on how the Partograph works and how it helps promote labour management and safe motherhood. 
Mwari P.S et.al. Assessment of the midwives knowledge on utilization of partograph in management of labour in public health facilities in Tharaka Nithi County.

Table 7: Midwives' Knowledge of Partograph Use

\begin{tabular}{|l|l|l|}
\hline Response & Frequency & Percentage \\
\hline Helps with safe motherhood implementation & 38 & 84.4 \\
\hline Enhances maternal death rate reduction & 40 & 88.9 \\
\hline Lowers the number of newborn deaths & 42 & 93.3 \\
\hline In normal progress of labour, the graph/plot on the Partograph should fall on the alert line & 28 & 51.1 \\
\hline $\begin{array}{l}\text { Provide plots that show labour progress and detect any anomalies with the plot falling on the left of } \\
\text { the alert line during a normal labor process }\end{array}$ & 12 & 26.7 \\
\hline The Partograph plot falls on the right of the alert line to indicate a normal labour progress & 40 & 88.9 \\
\hline Normal labour progress records 3 contractions for every 10 minutes & 30 & 66.7 \\
\hline
\end{tabular}

Beginning with the practices of Partograph utilization in Table 7, midwives expressed varying views on the implementation of the labor management tool in labor management. For example, $88.9 \%$ and $84.4 \%$ of midwives agreed that the Partograph helped reduce maternal deaths and improved safe motherhood, respectively. Another $93.3 \%$ saw Partograph as a tool that aided in the reduction of stillbirths.

The other points of view concerned the positioning of the Partograph plot when recording the labor process. From items 4 to 7 , respondents expressed a variety of opinions, as shown in Table 7. The results in Table 7 show inconsistencies in Partograph use among midwives, indicating incorrect use and insufficient knowledge.

\section{Function of the Action line on the Partograph}

The researcher went further to explore the functions of the action line on the Partograph from the midwives and obtained different views.

Table 8: Respondents' views about the function of the Action line on the Partograph

\begin{tabular}{|l|l|l|}
\hline Response & Frequency & Percentage \\
\hline Alert to take appropriate action & 42 & 93.3 \\
\hline $\begin{array}{l}\text { Gives midwives time to provide } \\
\text { adequate assessment for the most } \\
\text { appropriate intervention }\end{array}$ & 34 & 75.6 \\
\hline $\begin{array}{l}\text { Records progress on labour until } \\
\text { delivery }\end{array}$ & 38 & 84.4 \\
\hline
\end{tabular}

The majority of midwives (93.35 percent) thought the action line was used to alert the birth attendant to take appropriate action, while 75.6 percent thought its main role was to assess the labor progress and inform the midwives of the most appropriate intervention to use (Table 8). The results indicated that a significant percentage of respondents (84.4\%) believed that the Partograph was used in monitoring the entire labour process until delivery.

\section{Association between Midwives}

Knowledge and Utilization of Partograph

The study sought to establish the association between Midwives Knowledge and utilization of Partograph. The results are presented in Table 10.

Table 9: Chi-Square test on Midwives Knowledge on Utilization of Partograph and labour Management

\begin{tabular}{|l|l|l|l|}
\hline & Value & Df & $\begin{array}{l}\text { Assymp. Sig } \\
\text { (2 sided) }\end{array}$ \\
\hline Pearson Chi-square & $12.312^{\mathrm{a}}$ & 1 & 0.00 \\
\hline Likelihood ratio & 13.412 & 1 & 0.00 \\
\hline Linear by linear association & 12.017 & 1 & 0.00 \\
\hline N of Valid cases & 45 & & \\
\hline
\end{tabular}

The chi-square results of association between knowledge and utilization of the Partograph was significant $(p<0.05)$. The findings indicated that high knowledge levels were necessary for Partograph use in labor management in Tharaka Nithi County.

\section{DISCUSSION}

From the findings of this study, nearly three-quarters of TNC midwives (74\%) used Partograph to monitor labor. The 26\% who reported not using the labor management tool in their practice raised concerns about the safety of mothers and unborn babies in various maternity units throughout the County. These findings are similar to those of a Mexican study on assessing knowledge of Partograph utilization in midwifery and labor monitoring which reported that reported higher odds of operative deliveries and increased number of infants with low APGAR scores at 5 minutes among mothers who were assisted to deliver without the use Partograph. 
From the findings of the focus group, only specific midwives recorded and assessed labor using the Partograph. According to the mothers, some midwives would contact clinical officers and doctors for assistance in interpreting the Partograph recordings. These findings imply that public health facilities in TNC lag behind in terms of Partograph utilization and have not met the WHO's standards. More training on the use of Partograph is required for the Midwives.

Inconsistencies in the definition of a Partograph and its functions in labor management were reported by midwives. While some (84.4\%) thought the tool was a chart used by doctors to monitor labor, others (62.2\%) thought it was a simple graphic that records labor progress to alert midwives about any conditions that would put the mother or child at risk. Another 71.1 $\%$ thought a Partograph was a pictorial overview used by midwives in labor monitoring. The findings on Partograph functions are inconsistent, which is similar to the findings of a study conducted in Amhara Region, Ethiopia, to investigate midwives' literacy levels in Partograph filling and its components [13]. Both studies demonstrate the theory-practice gap that birth attendants face in labor units. Only one-quarter of respondents could comfortably identify and explain the constituents of a Partograph. The findings revealed disparities in Partograph utilization knowledge between trained and untrained midwives [13]

More than half of the midwives (60\%) had received training in the use of Partograph. Despite working in the maternity wings, $40 \%$ of them had not been trained on how to use the labor management tool. The WHO requires health facilities to invest in in-service training to ensure that all of their employees have up-to-date labor management skills. The findings mirrored the situation in TNC's public health facilities, where there was a significant theory-practice gap in Partograph use. According to the study, $96.6 \%$ of Ethiopian midwives were aware of the Partograph, but only a small percentage of them used it to monitor labor during delivery [6].

Findings on the importance of Partograph in labor management in reducing stillbirths and maternal deaths agrees with the findings of Cagayan about partograph's role in the management of obstetric emergencies and provides the most appropriate interventions to reduce global maternal deaths. However, the inconsistencies and differing perspectives on the functionality of the Partograph can similarly be explained as in study, which showed incorrect use of the Partograph [11].

The mismatch in Partograph utilization among midwives in labor management is explained by limited inservice training among midwives and a lack of advocacy on Partograph implementation. The disparities in midwives' perspectives on the functions of the action line in the Partograph indicate a knowledge gap in health workers regarding Partograph utilization. The knowledge of use of the Partograph deviates from the WHO's recommendation that the tool be used to identify the possibility of slow labor progress in the early stages and to prevent prolonged labor and related complications such as fistula, cesarean section, and ruptured uterus. The findings revealed a significant theory-practice gap in the implementation of Partograph utilization across Tharaka Nithi's public health facilities.

\section{CONCLUSION}

Tharaka Nithi public health facilities employ qualified midwives with the necessary academic credentials. The majority of midwives have more than five years of experience, with some having worked for more than 15 years. The majority of these midwives are aware of the use of Partograph in labor management, but some have never used the tool due to limited knowledge. However, even after receiving training in Partograph use, some midwives continue to use it incorrectly or rely on other 
health workers for recording and interpretation. In these public health facilities, there are very few midwives who can use the Partograph correctly, as recommended by the WHO. Within the county's health facilities, in-service training and policy on Partograph utilization are major challenges. Some midwives rely on their coworkers to teach them how to use the labor management tool, which should be a standard tool in labor wards. The study recommends additional research on the limitations of in-service training as well as policy frameworks for Partograph training and use in TNCs and the country as a whole.

\section{Competing Interests}

Authors have declared that no competing interest exists.

\section{Source of Funding: None}

\section{Ethical Approval: Approved}

\section{REFERENCES}

1. Taneja, G., Sarin, E., Bajpayee, D., Chaudhuri, S., Verma, G., Parashar, R., ... \& Gera, R. (2021). Care Around Birth Approach: A Training, Mentoring, and Quality Improvement Model to Optimize Intrapartum and Immediate Postpartum Quality of Care. Global Health: Science and Practice.

2. World Health Organization. (2014). Trends in maternal mortality: 1990 to 2013: estimates by WHO, UNICEF, UNFPA, The World Bank and the United Nations Population Division

3. World Health Organization, (2011). Department of Making Pregnancy Safer. WHO Partograph e-Learning Tool. World Health Organization, Geneva

4. Kibiwott, D., Mwangi, A., \& Kang'ethe, S. (2021). Partograph use among skilled birth attendants in selected counties, Western Kenya. International Journal of Nursing and Midwifery, 13(3), 19-25.

5. Mathibe-Neke, J. M., Lebeko, F. L., \& Motupa, B. (2013). The partograph: a labour management tool or a midwifery record?. International Journal of Nursing and Midwifery, 5(8), 145-153.

6. Yisma, E., Dessalegn, B., Astatkie, A., \& Fesseha, N. (2013).Knowledge and utilization of partograph among obstetric caregivers in public health institutions of Addis Ababa, Ethiopia. BMC pregnancy and childbirth, 13(1), 17.

7. Opiah, M. M., Ofi, A. B., Essien, E. J., \& Monjok, E. (2012).Knowledge and utilization of the partograph among midwives in the Niger Delta Region of Nigeria. African journal of reproductive health, 125-132.

8. Kalisa, R., Rulisa, S., van den Akker, T., \& van Roosmalen, J. (2019). Is prolonged labor managed adequately in rural Rwandan hospitals?. African journal of reproductive health, 23(2), 27-34.

9. Oladapo, O. T., Daniel, O. J., \& Olatunji, A. O. (2006).Knowledge and use of the partograph among healthcare personnel at the peripheral maternity centers in Nigeria. Journal of Obstetrics and Gynaecology, 26(6), 538-541.

10. Rotich, E., Maina, L., Njihia, A., \& Christensson, K. (2011). Evaluating partograph use at two main referral hospitals in Kenya. African Journal of Midwifery and Women's Health, 5(1), 21-24.

11. Qureshi, Z. P., Sekadde-Kigondu, C., \& Mutiso, S. M. (2010). Rapid assessment of partograph utilization in selected maternity units in Kenya. East African medical journal, 87(6), 235-241.

12. Creswell, J. W. (2012). Educational research: Planning, conducting, and evaluating quantitative and qualitative.

13. Abebe, F., Birhanu, D., Awoke, W., \& Ejigu, T. (2013). Assessment of knowledge and utilization of the partograph among health professionals in Amhara region, Ethiopia. Science journal of clinical medicine, 2(2), 26-42.

How to cite this article: Mwari P.S, Gitonga LK, Mukhwana E.S. Assessment of the midwives knowledge on utilization of partograph in management of labour in public health facilities in Tharaka Nithi County. Int J Health Sci Res. 2021; 11(12): 167-175. DOI: https://doi.org/10. 52403/ijhsr.20211223 